\title{
A Model for Jet-Surface Interaction Noise Using Physically Realizable Upstream Turbulence Conditions
}

\author{
Mohammed Z. Afsar ${ }^{1}$ \\ Imperial College London, 180 Queen's Gate, London, SW7, UK \\ S.J. Leib ${ }^{2}$ \\ Ohio Aerospace Institute, 22800 Cedar Point Road, Cleveland, Ohio 44142, USA \\ and \\ Richard F. Bozak ${ }^{3}$ \\ National Aeornautics and Space Administration, Glenn Research Center, Cleveland, Ohio 44135, USA
}

\begin{abstract}
This paper is a continuation of previous work in which a generalized Rapid Distortion Theory (RDT) formulation was used to model low-frequency trailing-edge noise. The research was motivated by proposed next-generation aircraft configurations where the exhaust system is tightly integrated with the airframe. Data from recent experiments at NASA on the interaction between high-Reynolds-number subsonic jet flows and an external flat plate showed that the power spectral density (PSD) of the far-field pressure underwent considerable amplification at low frequencies. For example, at the $\mathbf{9 0}^{\circ}$ observation angle, the low-frequency noise could be as much as $10 \mathrm{~dB}$ greater than the jet noise itself. In this paper, we present predictions of the noise generated by the interaction of a rectangular jet with the trailing edge of a semi-infinite flat plate. The calculations are based on a formula for the acoustic spectrum of this noise source derived from an exact formal solution of the linearized Euler equations involving (in this case) one arbitrary convected scalar quantity and a Rayleigh equation Green's function. A low-frequency asymptotic approximation for the Green's function based on a two-dimensional mean flow is used in the calculations along with a physically realizable upstream turbulence spectrum, which includes a finite de-correlation region. Numerical predictions, based on three-dimensional RANS solutions for a range of subsonic acoustic Mach number jets and nozzle aspect ratios are compared with experimental data. Comparisons of the RANS results with flow data are also presented for selected cases. We find that a finite decorrelation region increases the low-frequency algebraic decay (the low frequency "rolloff") of the acoustic spectrum with angular frequency thereby producing much closer agreement with noise data for Strouhal numbers less than 0.1. Secondly, the large-aspectratio theory is able to predict the low-frequency amplification due to the jet-edge interaction reasonably well, even for moderate aspect ratio nozzles. We show also that the noise predictions for smaller aspect ratio jets can be fine-tuned using the appropriate RANS-based mean flow and turbulence properties.
\end{abstract}

\footnotetext{
${ }^{1}$ Research Fellow, Member AIAA.

${ }^{2}$ Senior Scientist, Senior Member AIAA.

${ }^{3}$ Researcher, Member AIAA.
} 


\section{Nomenclature}

\begin{tabular}{|c|c|}
\hline$c$ & $=$ sound speed \\
\hline$c_{\infty}$ & $=$ ambient sound speed \\
\hline$D_{J}$ & $=$ nozzle diameter \\
\hline$g$ & $=$ Green's function \\
\hline$I_{\omega}$ & $=$ acoustic spectrum \\
\hline$k$ & $=$ turbulent kinetic energy \\
\hline$k_{1}$ & $=$ streamwise wavenumber \\
\hline$l_{i}$ & $=$ characteristic length scale \\
\hline$M$ & $=$ acoustic Mach number \\
\hline$M_{a}$ & $=$ jet acoustic Mach number \\
\hline$p$ & $=$ pressure \\
\hline$t$ & $=$ time \\
\hline$T$ & $=$ averaging time \\
\hline$U_{c}$ & $=$ convection velocity \\
\hline$V$ & $=$ source volume \\
\hline$v_{i}$ & $=$ velocity vector \\
\hline $\boldsymbol{x}$ & $=$ observer location \\
\hline$y$ & $=$ source location \\
\hline$\alpha$ & $=$ turbulence intensity \\
\hline$\beta$ & $=$ angle function \\
\hline$\gamma$ & $=$ specific heat ratio \\
\hline$\delta_{i j}$ & $=$ Kronecker delta \\
\hline$\varepsilon$ & $=$ turbulence dissipation rate \\
\hline$\eta$ & $=$ separation vector \\
\hline$\rho$ & $=$ density \\
\hline$\theta$ & $=$ polar angle measured from jet axis \\
\hline$\tau$ & $=$ time delay \\
\hline$\omega$ & $=$ radian frequency \\
\hline$\nabla$ & $=$ gradient operator \\
\hline $\mid$ & $=$ absolute value \\
\hline
\end{tabular}

Subscripts

$i, j, k, l=$ tensor indices $=1,2,3$

$\perp \quad=$ transverse component

Superscripts

$\begin{array}{ll}a & =\text { adjoint } \\ - & =\text { time average }\end{array}$ 
$=$ fluctuating quantity

$=$ Favre average

$=$ complex conjugate

\section{Introduction}

Jet flows of technological interest are almost always in close proximity to, or confined by, solid boundaries, such that the surface defining the boundary plays a direct role in the generation of sound and its propagation. Understanding the basic physics behind this process is of considerable importance for present-day and future aircraft that may have complex engine installation geometries. The aim of this paper is further develop a prediction method for the noise generated by the interaction of a turbulent jet with the trailing edge of a flat plate. This problem serves as a model of one important aspect of engine-installation effects, namely the interaction of the exhaust jet with a wing. The prediction method is based on a self-consistent application of the non-homogeneous Rapid-distortion theory (RDT) introduced recently in [1] (hereafter referred to as GAL).

Experiments conducted by Olsen \& Boldman [2] and Wang [3] showed that the presence of an external surface enhanced the noise produced by the jet alone for observation points on the same side as the jet flow. Recent experiments at the NASA Glenn Research Center ([4],[5],[6],[7],[8]) have considered the jet-wing interaction problem as a jet flow interacting with a trailing edge of an external plate. The power spectral density of the far-field pressure (PSD) was measured for unheated, high Reynolds number, jet flows across a range of acoustic Mach numbers when the trailing edge was positioned above/beneath the flow at various axial/radial locations relative to the nozzle center line.

The findings of [5] have generally confirmed the trends of [2] and [3]. In particular, at low frequencies, the PSD is considerably amplified compared to the free jet, and this effect is greatest at large polar observation angles to the jet axis (i.e. near $90^{\circ}$ ); see figures $6 \& 8$ in [5]. This low-frequency amplification effect has been called "jet-surface interaction" noise Brown [9], owing to its observed dipole characteristic and structural difference to high-frequency noise shielding and reflection. In [9] Brown, developed of an empirical model for jet-surface interaction noise, extracted the noise due to the jet/trailing edge interaction from measurements of the total noise in various jet-plate configurations using the expected dipole characteristics of the edge noise source.

In this paper we use the general theory developed in [1] to model the jet-surface interactions using RDT. Rapid Distortion Theory uses linear analysis to study the interaction of turbulence with, for example, solid surfaces. It applies whenever the turbulence intensity is small and the time scale for the interaction is short compared to the turn-over time of the turbulent eddies over which non-linear interaction and viscous dissipation take place Hence the basic equation of the problem is the compressible Rayleigh equation.

An initial application of the general theory to the jet-surface interaction problem was included in GAL. Here, we further develop the prediction method by introducing a more realistic model for the statistics of the upstream turbulence whose interaction with the plate edge generates noise. The modelling approach is similar to that in [12], but used here for the (second-order) transverse velocity auto covariance. In particular, we show that a region of negative correlation (or 'de-correlation') in this quantity directly impacts the low-frequency algebraic decay (often referred to as the 'roll-off') of the edge-generated noise and provides better agreement with experimental data than our previous results. The presence of such negative regions in second-order correlation has been known for some time ([13]). This result has implications for trailing-edge noise control.

The prediction capabilities of the method are further developed by using results from Reynolds-averaged NavierStokes (RANS) solutions to obtain the mean flow and inform the source model, in particular to obtain the turbulent length scales and source amplitude. The use of RANS solutions also allows these flow quantities to vary with flow conditions and geometry. The RANS solutions are obtained using the SolidWorks ${ }^{\circledR}$ Flow Simulation software ([10] [11]), which provides relatively fast solutions for the geometries of interest. As a check on the quality of these results, we include comparisons with measure flow data [6] for selected cases.

In the next section we summarize the main features of non-homogeneous RDT introduced in GAL and describe how this theory can be used for the trailing-edge noise problem. As mentioned above, there are two novel features of the present paper as compared to the trailing edge model in GAL. Firstly, we use a more advanced turbulence model for the two-point correlation of the transverse velocity fluctuations Secondly, noise predictions using results from RANS solutions to obtain the mean flow profile and turbulent kinetic energy (TKE) near the trailing edge are presented and compared with experimental data taken at NASA Glenn. 
In section II we briefly review the relevant parts of the GAL analysis used in this work. In section III, we introduce the new model for the transverse velocity correlations and present the corresponding formula for the acoustic spectrum. In section IV we illustrate some generic features of the model using an analytically prescribed mean flow. The impact of the new source model on the low-frequency "roll-off" of the spectrum and comparions with the model of GAL are shown. In section $\mathrm{V}$, the use of SolidWorks ${ }^{\circledR}$ software to obtain RANS solutions for a rectangular jet near the edge of flat plate is described. Results from these solutions are compared with experimental data taken at NASA Glenn [6] for the mean flow and turbulent kinetic energy distributions near the edge of the plate. In section VI we present comparisons of noise predictions using the model developed in this paper, with the SolidWorks RANS solutions as input, with data taken at NASA Glenn. Conclusions and discussion of potential future work is given in Section VII.

\section{Review of the GAL Formulation}

\section{A. Euler Equations for Small-Amplitude Motion}

Let all lengths and velocities be non-dimensionalized by $D_{J}$ and $U_{J}$, respectively; time by $U_{J} / D_{J}$ and pressure by $\rho_{J} U_{J}^{2}$, where $\rho_{J}$ and $U_{J}$ are flow density and velocity, respectively, at nozzle exit and $D_{J}$ is an appropriate reference length scale (such as the nozzle exit equivalent diameter). The flow Reynolds numbers is assumed to be large, ie. $\mathrm{R}=U_{J} D_{J} / v \gg O(1)$ and the turbulence Reynolds number is fixed at order 1 , i.e. $\mathrm{R}_{T}=\alpha \mathrm{R}=O(1)$; where $v$ is the kinematic viscosity and $\alpha \equiv\left|v^{\prime}\right| / U_{J} \ll O(1)$ is the turbulence intensity of the upstream flow. $\left|\boldsymbol{v}^{\prime}\right|$ is the magnitude of the local rms turbulence velocity.

We suppose that the flow is inviscid and non-heat conducting and assume an ideal gas so that the entropy is proportional to $\ln \left(p / \rho^{\gamma}\right)$ and the squared sound speed is $\gamma p / \rho$, where $p$ denotes the pressure, $\rho$ the density and $\gamma$ the specific heat ratio. Since the upstream entropy fluctuations are negligible, the inviscid pressure $p^{\prime}=p-p_{0}$ and momentum flux perturbations, $u_{i} \equiv \rho v_{\mathrm{i}}^{\prime}$, (where $v_{i}^{\prime}$ denotes the velocity perturbation) on a transversely sheared mean flow with pressure $p_{0}=$ constant, velocity $U\left(\boldsymbol{y}_{T}\right)$ and mean sound speed squared $c^{2}$, are governed by the linearized momentum and energy equations

$$
\frac{D_{0} u_{i}}{D \tau}+\delta_{1 i} u_{j} \frac{\partial U}{\partial y_{j}}+\frac{\partial p^{\prime}}{\partial y_{i}}=0
$$

and

$$
\frac{D_{0} p^{\prime}}{D \tau}+\frac{\partial c^{2} u_{j}}{\partial y_{j}}=0
$$

where, $\boldsymbol{y}=\left\{y_{1}, y_{2}, y_{3}\right\}=\left\{y_{1}, \boldsymbol{y}_{T}\right\} \quad, \boldsymbol{y}_{T}=\left\{y_{2}, y_{3}\right\}$ and $D_{0} / D \tau \equiv \partial / \partial \tau+U \partial / \partial y_{1}$ denotes the convective derivative.

1. Integration of the Euler equations (1) - (2) 
Goldstein [14] showed that equation (1) will be satisfied for any function $\phi(y, \tau)$ and any purely convected quantity, $\vartheta\left(\tau-y_{1} / U\left(\boldsymbol{y}_{T}\right), \boldsymbol{y}_{T}\right)$, when $p^{\prime}$ and $u_{i}$ are determined by

$$
p^{\prime}(\boldsymbol{y}, \tau)=-\frac{D_{0}^{3} \phi}{D \tau^{3}}(\boldsymbol{y}, \tau)
$$

and

$$
u_{i}(\boldsymbol{y}, \tau)=\left(\delta_{i j} \frac{D_{0}}{D \tau}-\delta_{i 1} \frac{\partial U}{\partial y_{j}}\right) \lambda_{j}(\boldsymbol{y}, \tau)+\varepsilon_{i j k} \frac{1}{c^{2}} \frac{\partial U}{\partial y_{j}} \frac{\partial}{\partial y_{k}} \vartheta\left(\tau-\frac{y_{1}}{U\left(\boldsymbol{y}_{T}\right)}, \boldsymbol{y}_{T}\right)
$$

where $\delta_{i j}$ denotes the Kronecker delta, $\varepsilon_{i j k}$ the alternating tensor and

$$
\lambda_{j} \equiv \frac{\partial}{\partial y_{j}} \frac{D_{0} \phi}{D \tau}+2 \frac{\partial U}{\partial y_{j}} \frac{\partial \phi}{\partial y_{1}}
$$

a kind of generalized particle displacement ([14], eqs. $2.9 \& 2.10)$.

The energy equation, (2), is then satisfied when the scalar $\phi(y, \tau)$ is determined by:

$$
\frac{D_{0}}{D \tau}\left[\frac{\partial}{\partial y_{i}} c^{2}\left(\frac{\partial}{\partial y_{i}} \frac{D_{0} \phi}{D \tau}+2 \frac{\partial U}{\partial y_{i}} \frac{\partial \phi}{\partial y_{1}}\right)-\frac{D_{0}^{3} \phi}{D \tau^{3}}\right]=0
$$

which can be integrated to obtain,

$$
L_{a} \phi=-\tilde{\omega}_{c}\left(\tau-y_{1} / U\left(\boldsymbol{y}_{T}\right), \boldsymbol{y}_{T}\right)
$$

where

$$
L_{a} \equiv \frac{D_{0}^{3}}{D \tau^{3}}-\frac{\partial}{\partial y_{i}} c^{2}\left(\frac{\partial}{\partial y_{i}} \frac{D_{0}}{D \tau}+2 \frac{\partial U}{\partial y_{i}} \frac{\partial}{\partial y_{1}}\right)
$$

and $\tilde{\boldsymbol{\omega}}_{c}\left(\tau-y_{1} / U\left(\boldsymbol{y}_{T}\right), \boldsymbol{y}_{T}\right)$ is another arbitrary, purely convected, quantity. GAL point out the operator $L_{a}$ is the adjoint to the Rayleigh operator,

$$
L \equiv \frac{D_{0}}{D \tau}\left(\frac{\partial}{\partial y_{i}} c^{2} \frac{\partial}{\partial y_{i}}-\frac{D_{0}^{2}}{D \tau^{2}}\right)-2 \frac{\partial U}{\partial y_{j}} \frac{\partial}{\partial y_{1}} c^{2} \frac{\partial}{\partial y_{j}}
$$

which is obtained by eliminating the momentum flux perturbation between (1) and (2) to derive a single equation for the fluctuating pressure

$$
L p^{\prime}=0
$$

The solution to $\phi(\boldsymbol{y}, \tau)$ can then be found by solving (7) as a boundary value problem in terms of the Rayleigh equation Green's function (as shown in GAL) 


$$
L g(\boldsymbol{y}, \tau \mid \boldsymbol{x}, t)=\delta(\boldsymbol{y}-\boldsymbol{x}) \delta(\tau-t),
$$

subject to appropriate boundary conditions, and the result inserted into (4) to obtain $u_{i}(\boldsymbol{y}, \tau)$.

\section{B. Integral Solutions to the Euler Equations for Arbitrary Transversely Sheared Mean Flows}

GAL showed that the formal complete solution to the non-homogeneous Rapid Distortion Theory problem for the pressure perturbation, $p^{\prime}$, is given by

$$
p^{\prime}(\boldsymbol{x}, t)=\int_{-T}^{T} \int_{V} \frac{D_{0}^{3} g(\boldsymbol{y}, \tau \mid \boldsymbol{x}, t)}{D t^{3}} \tilde{\boldsymbol{\omega}}_{c}\left(\tau-y_{1} / U\left(\boldsymbol{y}_{T}\right), \boldsymbol{y}_{T}\right) d \boldsymbol{y} d \tau
$$

(where $T$ denotes a very large but finite time interval) when the solid surfaces $S$ (which can be finite, semiinfinite or infinite in the streamwise direction) coincide with any level surfaces of the mean velocity profile (as in the trailing edge problem considered herein). The solution for $p^{\prime}$ in these cases is independent of the convected quantity $\vartheta\left(\tau-y_{1} / U\left(\boldsymbol{y}_{T}\right), \boldsymbol{y}_{T}\right)$.

The corresponding solution for the transverse momentum fluctuation is

$$
\begin{aligned}
\rho v_{\perp}^{\prime}(\boldsymbol{x}, t) & \equiv u_{i}(\boldsymbol{x}, t) \frac{\partial U}{\partial x_{i}} /|\nabla U| \\
& =-\frac{\partial U / \partial x_{i}}{|\nabla U|} \int_{-T}^{T} \int_{V} g_{i}(\boldsymbol{y}, \tau \mid \boldsymbol{x}, t) \tilde{\omega}_{c}\left(\tau-y_{1} / U\left(\boldsymbol{y}_{T}\right), \boldsymbol{y}_{T}\right) d \boldsymbol{y} d \tau
\end{aligned}
$$

where

$$
g_{i}(\boldsymbol{y}, \tau \mid \boldsymbol{x}, t) \equiv \frac{D_{0}}{D t}\left(\frac{\partial}{\partial x_{i}} \frac{D_{0}}{D t}+2 \frac{\partial U}{\partial x_{i}} \frac{\partial}{\partial x_{1}}\right) g(\boldsymbol{y}, \tau \mid \boldsymbol{x}, t)
$$

This solution involves only the purely convected quantity $\tilde{\omega}_{c}\left(\tau-y_{1} / U\left(\boldsymbol{y}_{T}\right), \boldsymbol{y}_{T}\right)$, that can be specified as an input condition within a general boundary value problem involving inhomogeneous boundary conditions. The Green's function $g(\boldsymbol{y}, \tau \mid \boldsymbol{x}, t)$ is found by solving (11) with incoming wave behavior as $|\boldsymbol{y}| \rightarrow \infty$ and appropriate boundary conditions (on the bounding surfaces $S$ that generate volume $V$ ) for (12) and (13) to hold.

\section{Green's Function Splitting}

Following GAL, we can think of solution (12) as being the sum of an input disturbance and downstream response (Figure 1). The trailing-edge noise (or the, output response) is generated by an input interacting with streamwise changes in boundary conditions at the plate edge. GAL divided the Rayleigh equation Green's function that appears in (12) into the components,

$$
g(\boldsymbol{y}, \tau \mid \boldsymbol{x}, t)=g^{(0)}(\boldsymbol{y}, \tau \mid \boldsymbol{x}, t)+g^{(s)}(\boldsymbol{y}, \tau \mid \boldsymbol{x}, t)
$$

where $g^{(0)}(\boldsymbol{y}, \tau \mid \boldsymbol{x}, t)$ is now defined on all space and satisfies 


$$
\hat{n}_{i} \partial\left[D_{0}^{3} g^{(0)}(\boldsymbol{y}, \tau \mid \boldsymbol{x}, t) / D t^{3}\right] / \partial y_{i}=0 \quad \text { for } \boldsymbol{y}_{T} \in S
$$

where $S$ is a doubly infinite surface and $\hat{\boldsymbol{n}}(\boldsymbol{y})=\left\{\hat{n}_{i}\right\}(\boldsymbol{y})$ is the unit outward-drawn normal to $S$.

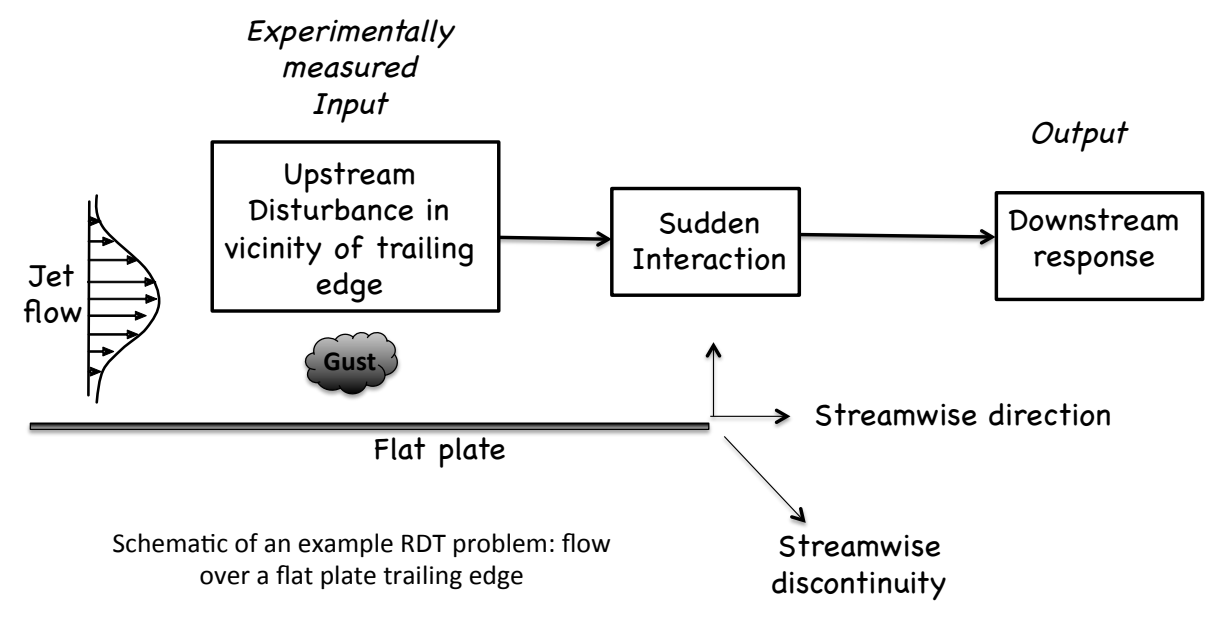

Figure 1 - Mathematical model of the jet-surface interaction noise.

Then, by (13), the corresponding transverse momentum flux is

$$
\rho v_{\perp}^{\prime}(\boldsymbol{x}, t)=-\frac{\partial U / \partial x_{i}}{|\nabla U|} \int_{-T}^{T} \int_{V} g_{i}^{(0)}(\boldsymbol{y}, \tau \mid \boldsymbol{x}, t) \tilde{\omega}_{c}\left(\tau-\frac{y_{1}}{U\left(\boldsymbol{y}_{T}\right)}, \boldsymbol{y}_{T}\right) d \boldsymbol{y} d \tau
$$

and then represents the input disturbance for the trailing-edge scattering problem and is referred to as the gust solution - i.e. a bounded hydrodynamic disturbance on a flow with streamwise homogeneous boundary conditions in the absence of any scattering surfaces. Although $\tilde{\omega}_{c}\left(\tau-y_{1} / U\left(\boldsymbol{y}_{T}\right), \boldsymbol{y}_{T}\right)$ is not a physically measurable quantitity, equation (17) provides an (in general) integral relation between $\tilde{\omega}_{c}\left(\tau-y_{1} / U\left(\boldsymbol{y}_{T}\right), \boldsymbol{y}_{T}\right)$ and the physical variable, $\rho v_{\perp}^{\prime}(\boldsymbol{x}, t)$ that can, in principle, be inverted (using identity (1.97) in Goldstein ([15]) to solve) for the Fourier transform of $\tilde{\omega}_{c}\left(\tau-y_{1} / U\left(\boldsymbol{y}_{T}\right), \boldsymbol{y}_{T}\right)$ in terms of the Fourier transform of $\rho v_{\perp}^{\prime}(\boldsymbol{x}, t)$.

\section{Relation Between the $\tilde{\omega}_{c}$ Spectrum and Measurable Turbulence Statistics}

The correspondence our input gust (17) has to the actual upstream turbulence in a "real" trailing-edge noise problem can be reconciled as follows. We are assuming the relation between $\tilde{\omega}_{c}\left(\tau-y_{1} / U\left(\boldsymbol{y}_{T}\right), \boldsymbol{y}_{T}\right)$ and $\rho v_{\perp}^{\prime}(\boldsymbol{x}, t)$ in the actual flow is the same as it would in an idealized mathematical representation of a transversely sheared mean 
flow where $\mathrm{R}_{T}=\alpha \mathrm{R}=O(1)$ everywhere in the flow and in which bounding surfaces present are doubly infinite in the streamwise direction (i.e. where the transverse boundary conditions are completely uniform in $y_{1}$ ). Hence the upstream boundary condition is determined on this streamwise homogeneous flow is assumed to be the same as that in the vicinity of trailing-edge where the "real" distortion actually takes place.

GAL showed that the inversion of (17) can be obtained analytically for a two-dimensional mean flow by computing the (temporal, streamwise and spanwise) Fourier transform of $\rho v_{\perp}^{\prime}(\boldsymbol{x}, t)$ and relating it exactly to the Fourier transform of $\tilde{\omega}_{c}\left(\tau-y_{1} / U\left(\boldsymbol{y}_{T}\right), \boldsymbol{y}_{T}\right)$, but that this relation can only be specified at $N$ discrete transverse space points, say $x_{2}^{(1)}, x_{2}^{(2)}, \ldots, x_{2}^{(N)}$, where $x_{2}^{(n)}$ is given by solutions to discrete equation, $U\left(x_{2}^{(n)}\right)=U\left(x_{2}\right)$, for $n=1,2, \ldots N$, leading to a matrix problem to determine the auto-covariance of the transform of $\tilde{\boldsymbol{\omega}}_{c}\left(\tau-y_{1} / U\left(\boldsymbol{y}_{T}\right), \boldsymbol{y}_{T}\right)$ in terms of the auto-covariance of the transform of $\rho v_{\perp}^{\prime}(\boldsymbol{x}, t)$. For jet flows, such as those considered in the present paper, there are two such points and, following GAL, we take $\left(x_{2}^{(1)}, x_{2}^{(2)}\right)=y_{d}$, where $y_{d}$ is the location where the velocity profile is maximum. In some sense, this gives an upper bound for the upstream boundary condition and allows the role of the de-correlation region to be assessed easily.

GAL used a uniformly valid low-frequency asymptotic solution for the gust Green's function in the relation between the Fourier transforms of $\rho v_{\perp}^{\prime}(\boldsymbol{x}, t)$ and $\tilde{\omega}_{c}\left(\tau-y_{1} / U\left(\boldsymbol{y}_{T}\right), \boldsymbol{y}_{T}\right)$ to obtain a relatively simple working formula relating the spectrum of the convected quantity $\tilde{\boldsymbol{\omega}}_{c}\left(\tau-y_{1} / U\left(\boldsymbol{y}_{T}\right), \boldsymbol{y}_{T}\right), S\left(y_{2}, \tilde{y}_{2} ; k_{3}^{(s)}, \omega\right)$,

$$
S\left(y_{2}, \tilde{y}_{2} ; k_{3}, \omega\right) \equiv \frac{1}{2 \pi} \int_{-\infty}^{\infty} \int_{-\infty}^{\infty} e^{i\left(\omega \tau-k_{3} \eta_{3}\right)}\left\langle\tilde{\omega}_{c}\left(t, y_{2}, y_{3}\right) \tilde{\omega}_{c}\left(t+\tau, \tilde{y}_{2}, y_{3}+\eta_{3}\right)\right\rangle d \tau d \eta_{3},
$$

to the experimentally measurable transverse velocity spectrum

$$
F_{\perp}\left(x_{2}, \tilde{x}_{2} \mid y_{2}, \tilde{y}_{2}, \omega, k_{3}\right)=\frac{1}{(2 \pi)^{2}} \int_{-\infty}^{\infty} \int_{-\infty}^{\infty} e^{-i\left(\omega \tau-k_{3} \eta_{3}\right)} f_{\perp}\left(x_{2}, \tilde{x}_{2} \mid \omega / U\left(y_{2}\right), \omega / U\left(\tilde{y}_{2}\right), \eta_{3}, \tau\right) d \eta_{3} d \tau
$$

where

$$
f_{\perp}\left(x_{2}, \tilde{x}_{2} \mid k_{1}, \tilde{k}_{1}, \eta_{3}, \tau\right) \equiv \frac{1}{(2 \pi)^{2}} \int_{-\infty}^{\infty} \int_{-\infty}^{\infty} e^{-i\left(x_{1} k_{1}-\tilde{x}_{1} \tilde{k}_{1}\right)}\left\langle\rho v_{\perp}^{\prime(0)}(\boldsymbol{x}, t) \rho v_{\perp}^{(0)}\left(\tilde{x}_{1}, \tilde{x}_{2}, x_{3}+\eta_{3}, t+\tau\right)\right\rangle d x_{1} d \tilde{x}_{1},
$$

as,

$$
S\left(y_{2}, \tilde{y}_{2} ; k_{3}, \omega\right)=\frac{\left|U^{\prime}\left(y_{2}\right) U^{\prime}\left(\tilde{y}_{2}\right)\right|}{\left[U\left(y_{2}\right) U\left(\tilde{y}_{2}\right)\right]} \frac{\left(1+\frac{y_{2}-y_{d}}{y_{d}} b_{0}\right)\left(1+\frac{\tilde{y}_{2}-y_{d}}{y_{d}} b_{0}\right) F_{\perp}\left(y_{d}, y_{d} \mid y_{2}, \tilde{y}_{2}, \omega, k_{3}\right)}{E\left(y_{2} ; k_{3}, \omega\right)\left[E\left(\tilde{y}_{2} ; k_{3}, \omega\right)\right]^{*}}
$$

where 


$$
E\left(y_{2} ; k_{3}, \omega\right) \equiv \frac{\left[U\left(y_{d}\right)-U\left(y_{2}\right)\right]}{c^{2}\left(y_{d}\right)}\left(1-\frac{\left|y_{2}-y_{d}\right|}{y_{d}} b_{0}\right)+\frac{\left[\omega^{2} / U^{2}\left(y_{2}\right)+k_{3}^{2}\right]}{\sqrt{\omega^{2} / U^{2}\left(y_{2}\right)+k_{3}^{2}-k_{\infty}^{2}}} \frac{i \pi U^{2}\left(y_{2}\right) b_{0}}{c_{\infty}^{2} U^{\prime \prime} y_{d}}
$$

$b_{0}$ is a constant relating the even and odd symmetry components of the Fourier transform of $\tilde{\omega}_{c}$ (see GAL) and $U^{\prime \prime}\left(y_{d}\right)$ is the second derivative of the mean velocity profile at $y_{d}$. We also use this formula for the calculations in this paper.

\section{E. Scattered Solution}

The scattered solution, $g^{(s)}(\boldsymbol{y}, \tau \mid \boldsymbol{x}, t)$ in the split formula (15), on the other hand, represents the contribution due to presence of the trailing edge and will satisfy streamwise inhomogeneous boundary and jump conditions on the streamwise-discontinuous surface present in the flow.

Imposing appropriate boundary/jump conditions on plate surface and its downstream extension leads to a Wiener-Hopf problem (eqs. 6.6-6.8 in GAL) for the (temporal, streamwise and spanwise) Fourier transform of $g^{(s)}(\boldsymbol{y}, \tau \mid \boldsymbol{x}, t)$. The general solution to this problem was derived by GAL, but since experiments show that the interaction of a turbulent jet and a trailing edge generates noise at relatively low frequencies, the analysis and computations were simplified by considering the low-frequency limit $\left(k_{1}, k_{3}\right)=O\left(k_{\infty}\right) \& k_{\infty} \ll O(1)$ for $y_{2}=O(1)$. This low-frequency approximation is also adopted in this paper.

\section{F. Acoustic spectrum formula for jet-surface interaction in planar flows}

For a two-dimensional jet with a planar mean flow, GAL showed that the acoustic spectrum for jet-surface interaction noise is given by remarkably simple asymptotic result,

$$
I_{\omega}(\boldsymbol{x}) \equiv \frac{1}{2 \pi} \int_{-\infty}^{\infty} e^{i \omega \tau} \overline{p^{s}(\boldsymbol{x}, t) p^{s}(\boldsymbol{x}, t+\tau)} d \tau \approx\left(\frac{k_{\infty}}{4 \pi|\boldsymbol{x}|}\right)^{2} \int_{0}^{\infty} \int_{0}^{\infty} D\left(\theta, \psi, M\left(y_{2}\right)\right) S\left(y_{2}, \tilde{y}_{2} ; k_{3}^{(s)}, \omega\right) d y_{2} d \tilde{y}_{2}
$$

in which the integrand can be interpreted as the product of the source function $S\left(y_{2}, \tilde{y}_{2} ; k_{3}^{(s)}, \omega\right)$ with a nonuniform directivity factor, $D\left(\theta, \psi, M\left(y_{2}\right)\right)$, that encapulates all propagation and surface interaction effects when $k_{\infty} \ll O(1)$ and $|\boldsymbol{x}| \rightarrow \infty . M\left(y_{2}\right)=U\left(y_{2}\right) / c_{\infty}$ denotes the local acoustic Mach number at the position $y_{2}$ and the spanwise wavenumber $k_{3}^{(s)}=k_{\infty} \sin \theta \cos \psi$ is found by applying the method of stationary phase for the inverse Fourier integral in $k_{3}$ (see GAL, p. 553). Here, $\theta$ is the polar observation angle measured relative to the jet center line and $\psi$ is the azimuthal angle in the cross-stream plane.

The directivity factor, given by

$$
D\left(\theta, \psi, M\left(y_{2}\right)\right)=\frac{\left[M\left(y_{2}\right) M\left(\tilde{y}_{2}\right)\right]^{3 / 2}(\beta-\cos \theta)}{\left[1-M\left(y_{2}\right) \cos \theta\right]\left[1-M\left(\tilde{y}_{2}\right) \cos \theta\right] \sqrt{\left[1-\beta M\left(y_{2}\right)\right]\left[1-\beta M\left(\tilde{y}_{2}\right)\right]}},
$$


and reduces to Goldstein's ([14], eq. 3.23) result $\sin ^{2} \frac{\theta}{2} /\left[1-M\left(y_{d}\right) \cos \theta\right]^{2}$ at the point where the velocity profile is maximum $y_{2}=\tilde{y}_{2}=y_{d}$ since $\beta \equiv\left(1-\sin ^{2} \theta \cos ^{2} \psi\right)^{1 / 2}$, is unity at $\psi= \pm \pi$, in the plane perpendicular to the plate. In Figure 2 we show that $D(\theta, \psi, M a)$ peaks at $90^{\circ}$ for subsonic acoustic Mach numbers. The azimuthal variation of $D(\theta, \psi, M a)$ possesses dipole-like structure at any point $y_{2}=\tilde{y}_{2}$ (see figure 4.1b).
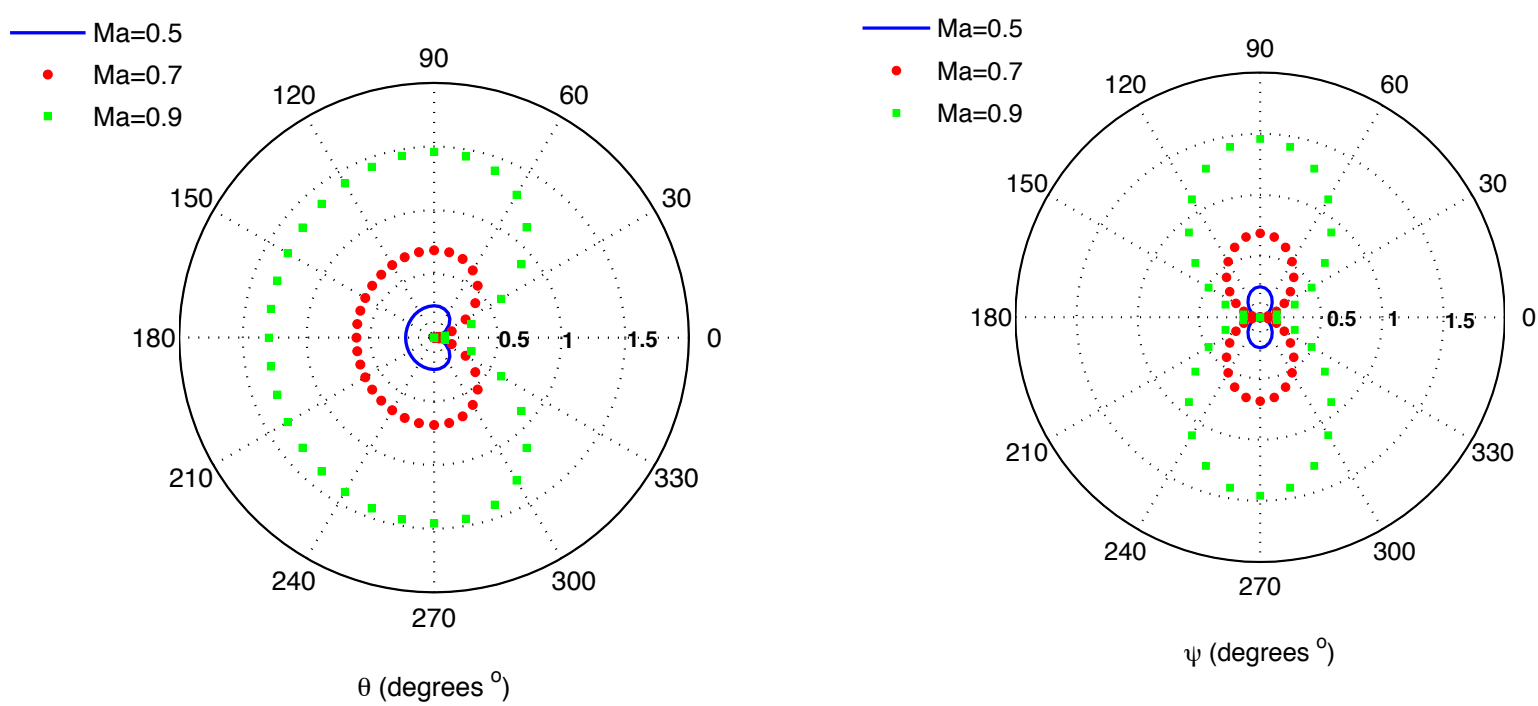

Figure 2 - Directivity factor: (a) $\theta$-directivity, (b) $\psi$-directivity

\section{Physically Realizable Upstream Turbulence Conditions}

\section{A. Turbulence Model}

The jet-surface interaction noise model (23) was constructed for a two-dimensional jet with planar mean flow. Consistent with this approximation, we suppose that the turbulence is spanwise homogeneous. The space-time average

$$
\left\langle v_{\perp}^{\prime}(\boldsymbol{x}, t) v_{\perp}^{\prime}\left(x_{1}, \tilde{x}_{2}, x_{3}+\eta_{3}, t+\tau\right)\right\rangle \equiv \lim _{T \rightarrow \infty} \frac{1}{2 T} \int_{-T}^{T} \int_{-\infty}^{\infty} v_{\perp}^{\prime}(\boldsymbol{x}, t) v_{\perp}^{\prime}\left(x_{1}, \tilde{x}_{2}, x_{3}+\eta_{3}, t+\tau\right) d t d x_{3}
$$

that enters the integrand of (20) is both experimentally determinable and has a well established database (e.g. experiments reported in [16]).

In this section, we construct a model for this function that is physically realizable and use it to derive a formula for the acoustic spectrum of the trailing edge noise. Different from GAL, however, the present model is includes a finite de-correlation region, which we show has a direct impact on the jet surface interaction noise and, in principle, could provide a means to reduce it. 
We use the function ([12]):

$$
\begin{aligned}
& \left\langle\rho v_{\perp}^{\prime}\left(x_{1}, y_{d}, x_{3}, t\right) \rho v_{\perp}^{\prime}\left(\tilde{x}_{1}, y_{d}, x_{3}+\eta_{3}, t+\tau\right)\right\rangle= \\
& L_{3} \Psi\left(\bar{x}_{1}\right)\left[a_{0}+a_{1} \tau \frac{\partial}{\partial \tau}+a_{2} \eta_{1} \frac{\partial}{\partial \eta_{1}}+\ldots\right] e^{-X\left(\tau, \eta_{1}, \eta_{3}\right)}
\end{aligned}
$$

where the decay function is,

$$
X\left(\eta_{1}, \eta_{3}, \tau\right)=\sqrt{\left(\eta_{1} / l_{1}\right)^{2}+\left(\eta_{1}-U_{c} \tau\right)^{2} / l_{0}^{2}+\left(\eta_{3} / l_{3}\right)^{2}}
$$

and the streamwise and spanwise separations are $\eta_{1} \equiv \tilde{x}_{1}-x_{1}$ and $\eta_{3}$, respectively. $l_{1}$ and $l_{3}$ are turbulence length scales in streamwise and spanwise directions, respectively; $l_{0}$, on the other hand, measures the scale of turbulence as it convects over separation distance $\eta_{1}$ with convection velocity, $U_{c}$.

Following GAL we allow $\Psi$ to decay in the streamwise direction in order to insure convergence of the subsequent Fourier transform integrals, i.e. $\Psi\left(\bar{x}_{1}\right)=\Psi_{0} e^{-\alpha\left(\bar{x}_{1} / L_{1}\right)^{2}}$ where $\alpha$ is a small positive number, $0 \leq \alpha<1$ which depends on the symmetric location $\bar{x}_{1} \equiv\left(x_{1}+\tilde{x}_{1}\right) / 2$, since we this quantity is expected to be independent of the streamwise coordinate for the nearly parallel flow being considered. $\Psi_{0}$ is expected to scale with the transverse component of the mean square turbulence momentum flux $\overline{\left(\rho v_{\perp}^{\prime}\right)^{2}}, L_{1}, L_{3}$ are geometric spatial scales: $L_{3}$ being a measure of the transverse extent of the turbulence and the $L_{1}$, is taken to be large in order to insure that the correlation (26) is relatively independent of $\bar{x}_{1}$ to remain consistent with our representation of the upstream boundary condition described in section II.

As shown in Figure 3, allowing the coefficient $a_{1}>0$ in equation (26) gives a negative (de-correlation) region for the auto-correlation $\left(\tau=\eta_{3}=0\right)$ function of (26) which did not appear in the model used in GAL (eq. 6.46). As mentioned in the Introduction, the presence of this negative region is an expected characteristic of the second-order transverse velocity correlations.
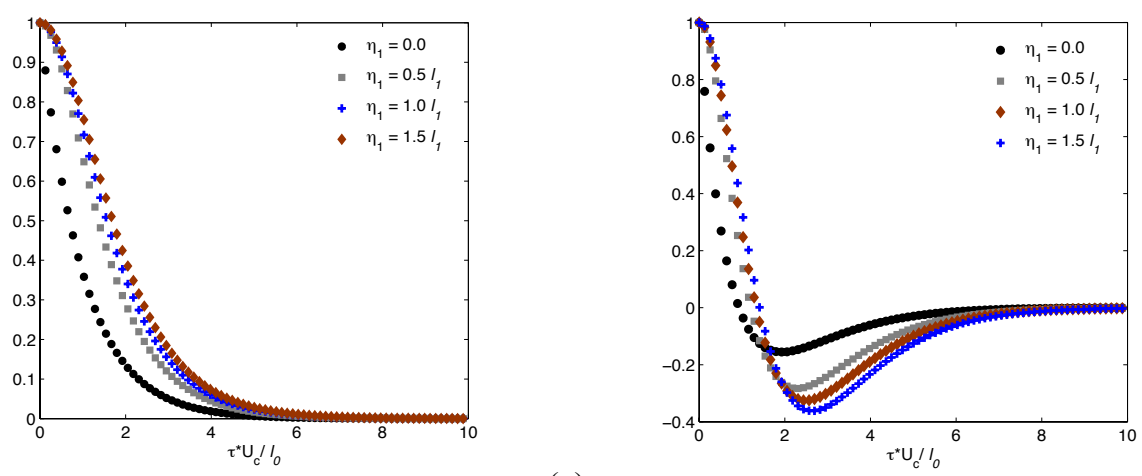

(a)

Figure 3 - Turbulence model (26) with (27) for $\tau=\eta_{3}=0$. (a) GAL model $a_{1}=a_{2}=0$;

(b) Present Model model $a_{1}>0$. 


\section{B. Acoustic spectrum formula with a finite de-correlation region}

Substituting the source model function (26) in (18)-(20) for $S\left(y_{2}, \tilde{y}_{2} ; k_{3}^{(s)}, \omega\right)$ and inserting this result in (23), we obtain the final formula for the acoustic spectrum used in this paper

$$
I_{\omega}(\boldsymbol{x})=\left(\frac{1}{4 \pi|\boldsymbol{x}|}\right)^{2}\left(\frac{1}{\pi^{2}}\right) \frac{l_{0} l_{1} l_{3}}{U_{c}}\left(\frac{L_{3} \Psi_{0} k_{\infty}}{c_{\infty}}\right)(\beta-\cos \theta) I_{0}(M a, \theta, \psi)
$$

where $\beta$ is defined below (24) and

$$
I_{0}(M a, \theta, \psi)=4 \int_{0}^{M a} \frac{c_{\infty} M\left(y_{2}\right)^{3}}{\left[1-M\left(y_{2}\right) \cos \theta\right]^{2}} \frac{\tilde{\Pi}\left(\omega / U\left(y_{2}\right), \omega, k_{3}^{(s)}\right)}{\left|E\left(y_{2} ; k_{3}^{(s)}, \omega\right)\right|^{2}\left[1-\beta M\left(y_{2}\right)\right]} d M\left(y_{2}\right)
$$

where $M a \equiv U\left(y_{d}\right) / c_{\infty}$ denotes the maximum acoustic Mach number and spectral functions $E\left(y_{2} ; k_{3}^{(s)}, \omega\right)$ is given by (22) and $\widetilde{\Pi}\left(\omega / U\left(y_{2}\right), \omega, k_{3}^{(s)}\right)$ is defined by:

$$
\begin{aligned}
& \tilde{\Pi}\left(k_{1}, \omega, k_{3}\right)= \\
& \frac{1}{\left(\left(\omega l_{0} / U_{c}\right)^{2}+\chi\right)^{2}}\left[\left(a_{0}-a_{1}-a_{2}\right)+\frac{4}{\left(\left(\omega l_{0} / U_{c}\right)^{2}+\chi\right)}\left[a_{1}\left(\frac{\omega l_{0}}{U_{c}}\right)^{2}-\left(a_{2} k_{1}+a_{1} \frac{\omega}{U_{c}}\right)\left(k_{1}-\omega / U_{c}\right) l_{1}^{2}\right]\right]
\end{aligned}
$$

where

$$
\chi=\chi\left(k_{1}, k_{3}\right)=\left[\left(k_{3} l_{3}\right)^{2}+\left(k_{1}-\omega / U_{c}\right)^{2} l_{1}^{2}+1\right] .
$$

The derivation of this formula is summarized in appendix A of Afsar et al. [17].

\section{Low-Frequency Roll-Off}

The predictions in GAL were compared against the jet-surface interaction experiments performed at NASA Glenn ([5],[6],[7],[8],[9]). The relevant geometric parameters are shown in Figure 4 


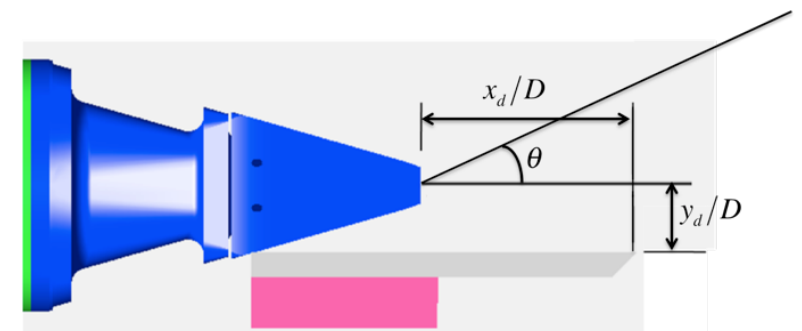

Figure 4 - Nozzle/plate configuration. Figure courtesy Dr. James E. Bridges, NASA Glenn

However it is clear from figure 6 in GAL, that for Strouhal numbers less than 0.15 (where the peak noise amplification occurs), the positive auto-correlation model (i.e. $a_{1}=a_{2}=0$ in (26)) they used over predicts the the low frequency roll-off for jet-surface interaction noise by as much as $10 \mathrm{~dB}$ (see figure $6 \mathrm{~d}$ in GAL).

We can easily prove why this occurs by estimating integral $I_{0}(M a, \theta, \psi)$ defined by (29), for very small frequencies. As $\omega \rightarrow 0$, the dominant contribution to integral comes in the vicinity of critical level $y_{2}=O\left(y_{d}\right)$. In the appendix, we estimate terms in (29) under this limit by consdering $\psi= \pm 90^{\circ}$ (which is the azimuthal location of the data for which the GAL predictions were made); $\beta=1$ and $k_{3}^{(s)}=0$ here. Substituting equation asymptotic properties (38) into acoustic spectrum formula (28) shows that the latter possesses asymptotic properties,

$$
I_{\omega}(\boldsymbol{x})=\left\{\begin{array}{l}
O(1), \text { for } a_{1}=a_{2}=0 \\
O\left(\omega^{2}\right), \text { for } a_{0} \sim a_{1}
\end{array}\right.
$$

when $\omega \rightarrow 0$ that is now directly dependent on the whether a de-correlation region in (26) exists or not.

In the absence of a de-correlation region in (26) (i.e. $a_{1}=a_{2}=0$ ) the acoustic spectrum does not possess a low frequency roll-off as such and tends to $O(1)$ (i.e. a constant, see equation (6.52) in GAL). A finite de-correlation region, however, increases the low frequency 'roll-off' in the prediction of (28) to exhibit an asympotic order of $O\left(\omega^{2}\right)$ or so, which is more dipole-like and appears to be more consistent with the experimental data. This is shown below in Figure 5 by comparing against experimental data measured at the shielded location with microphone array below plate ([8]) and for a mean velocity profile (eq. 6.55) used in GAL. 


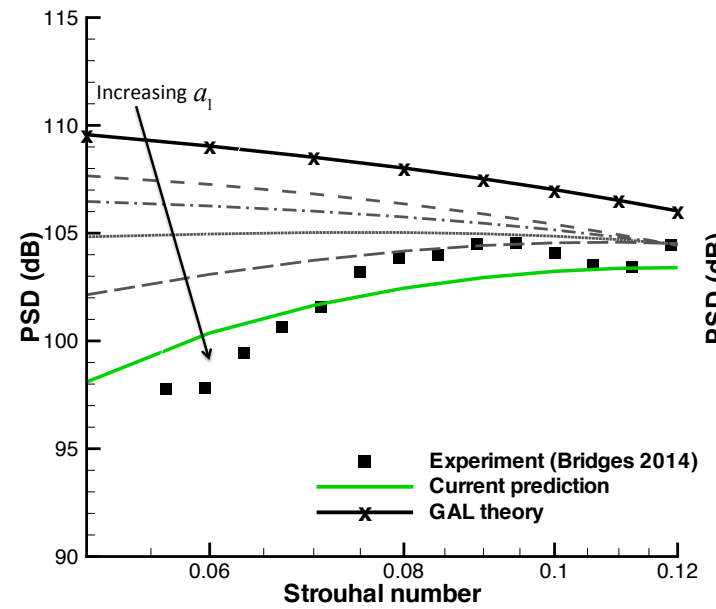

(a). Parametric increase in $a_{1}$ with $\left(a_{0}=1 ; a_{2}=0\right)$.

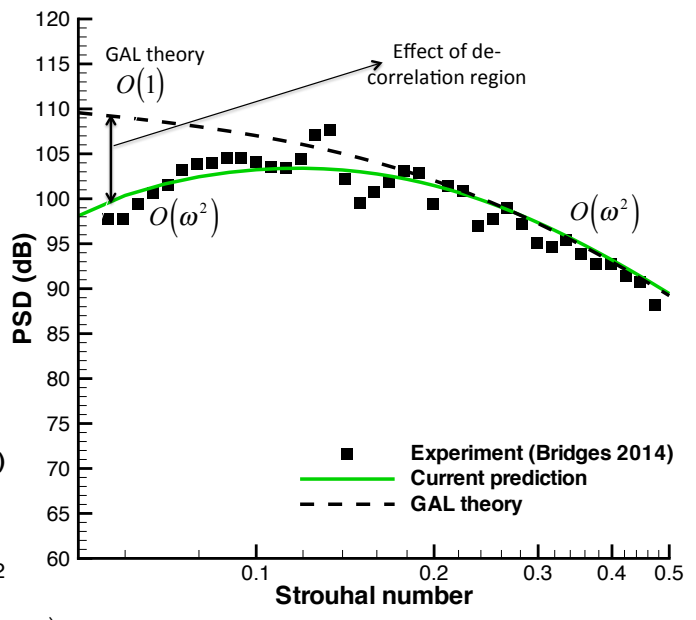

(b). Equation (28) using model (26).

Figure 5 - Power Spectral Density (PSD) of the far-field pressure fluctuations at 100 equivalent diameters from nozzle exit (lossless in $\mathrm{dB}$ scale referenced to $20 \mu \mathrm{Pa}$ ) as a function of Strouhal number, for $M a=0.9$.

Plate trailing edge at $y_{d} / D_{J}=1.2, x_{d} / D_{J}=5.7, D_{J}=2.12^{\prime \prime}, \psi= \pm 90^{\circ}$ and $\theta=90^{\circ}$. Source model constants for GAL theory are the same as their figure 4. Source model constants for current predictions are $\Psi_{0}=0.04\left(\rho_{\infty} U_{d}\right)^{2} ;\left(l_{0}, l_{1}, l_{3}\right) / D_{J}=(0.53,0.01,0.01) ;\left(L_{2}, L_{3}\right) / D_{J}=(0.5,20), \quad U_{c}=0.68 U_{d} ; b_{0}=0.52 \quad$ and $\left(a_{0}, a_{1}, a_{2}\right)=(0.82,0.88,0.05)$.

In Figure 6 we show that the acoustic spectrum (28) possesses a dipole-like azimuthal structure. Here we consider the polar angle of $90^{\circ}$ where the jet-surface interaction noise is greatest and a peak Strouhal number which looking at Figure 5 occurs at about $\mathrm{St}=0.12$. The turbulence, defined through the source term $S\left(y_{2}, \tilde{y}_{2} ; k_{3}^{(s)}, \omega\right)$, amplifies the pure propagation effects described through the directivity factor shown in Figure 2.

Figure 7 shows that even though the GAL theory is based on a two-dimensional mean flow (i.e. on a large aspect ratio rectangular jet nozzle) it provides a reasonable estimate to the lower aspect ratio (AR) jet-surface interaction noise. 


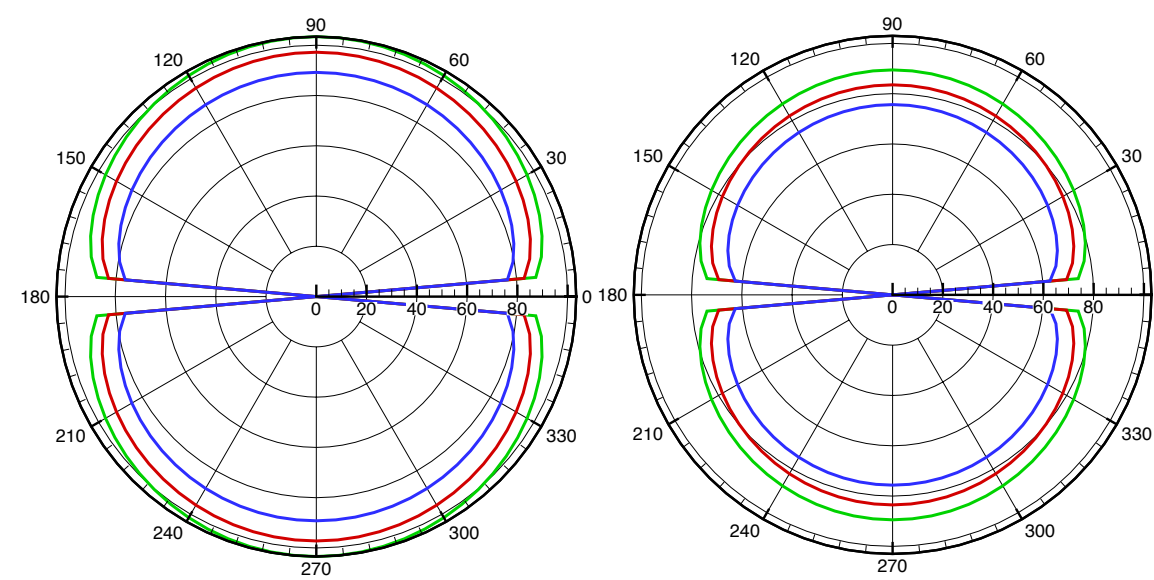

Figure 6 - $\psi$-directivity of RDT prediction (28) at peak Strouhal number (St) and upper limit as shown in Figure 5 for $M\left(y_{2}\right)=M a=(0.5,0.7,0.9)$ (colour coding same as Figure 2). Source model constants for RDT prediction is same as Figure 5.

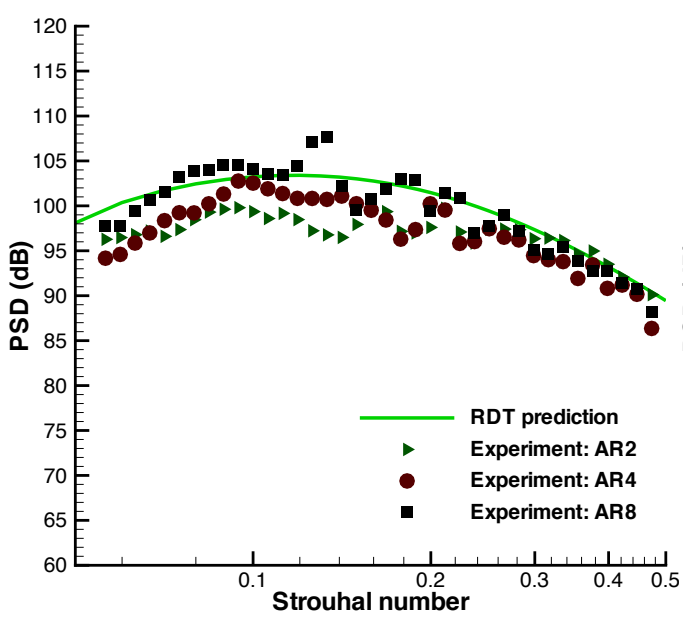

(a). $M a=0.9$ and $\theta=90^{\circ}$.

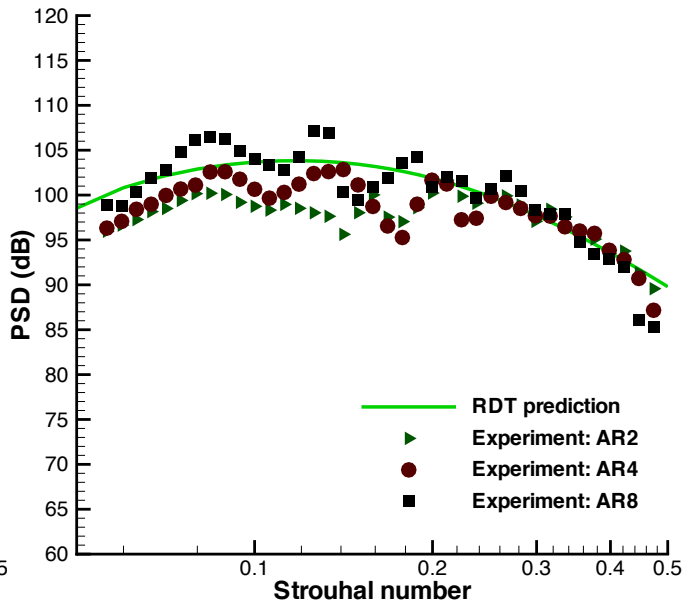

(b). $M a=0.9$ and $\theta=75^{\circ}$ 


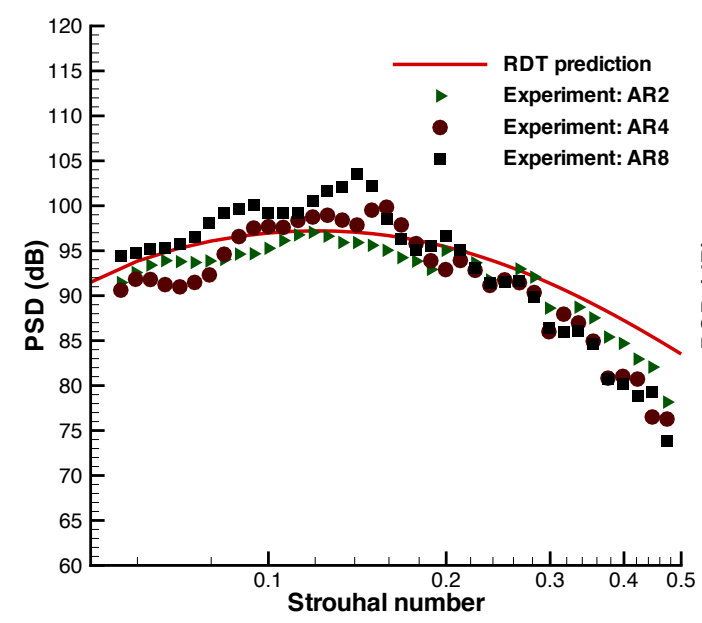

(c). $M a=0.7$ and $\theta=90^{\circ}$.

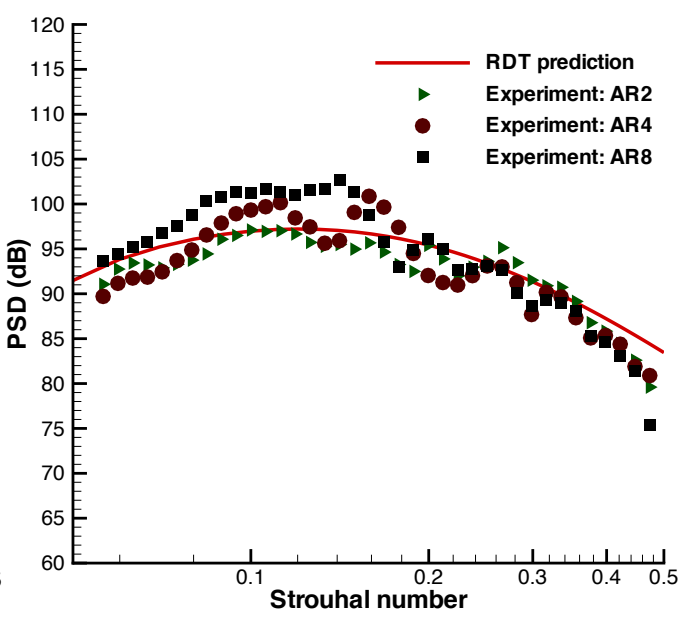

(d). $M a=0.7$ and $\theta=75^{\circ}$

Figure 7 - RDT predictions compared to noise data from various nozzle aspect ratio (AR) rectangular jets. Source model constants and mean flow for RDT prediction is same as Figure 5.

\section{RANS Solutions}

Although the RDT predictions based on an analytical mean flow shown in Figure 7 are in reasonable agreement with data, it is expected that the turbulence length scales and peak turbulent kinetic energy (TKE) levels near the trailing edge will change with nozzle aspect ratio and flow conditions. These variations can be accommodated within the RDT model using a RANS-based mean flow, TKE and rate of energy dissipation $(\varepsilon)$ to define the mean velocity profile, length scales and amplitude of the source function, $S$, near the trailing edge.

In this section we show results from three-dimensional SolidWorks ${ }^{\circledR}$ Flow Simulations of jet flows with acoustic Mach numbers, $M a=(0.5,0.7,0.9)$ through rectangular jet nozzles with aspect ratios, $A R=(2,4,8)$. The SolidWorks ${ }^{\circledR}$ Flow Simulation automatic gridding methodology ([10],[11]) provides a way to mesh and solve the flow field around complex geometries and is, therefore, rather convenient for the jet surface interaction problem. In Figure 8, mean axial velocity and turbulent kinetic energy RANS results are compared with hotwire data from Zaman et al. [6]. The results are compared for the same aspect ratio (8) and surface length (12-inches), but, owing to facility constraints, at different flow conditions and very slightly different surface offsets. The RANS results have a surface offset of $y_{2} / D_{J}=1.05$, whereas the offset in the experiment is $y_{2} / D_{J}=1.0$. In comparing the RANS and hot-wire data, we have normalized the mean axial velocity and turbulent kinetic energy using the ideally-expanded jet exit velocity as, $U_{J}$ and $U_{J}^{2}$, respectively. It is expected that these normalized results can be reasonably compared to give some idea of the validity of the SolidWorks ${ }^{\circledR}$ RANS solutions. Further comparisons are planned once more data becomes available. 


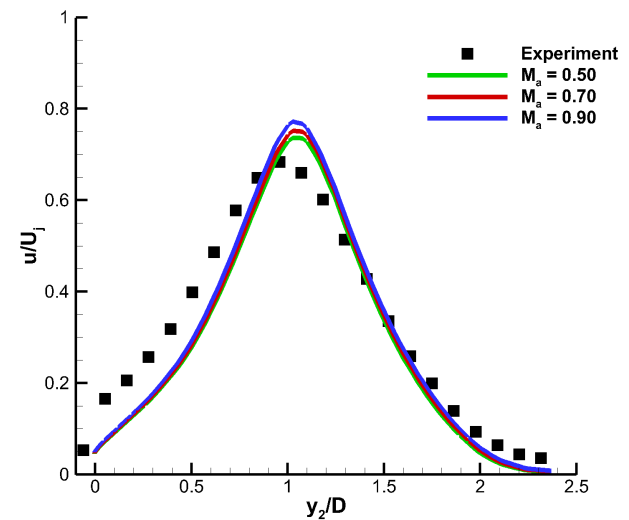

(a). Mean velocity, $U / U_{J}$

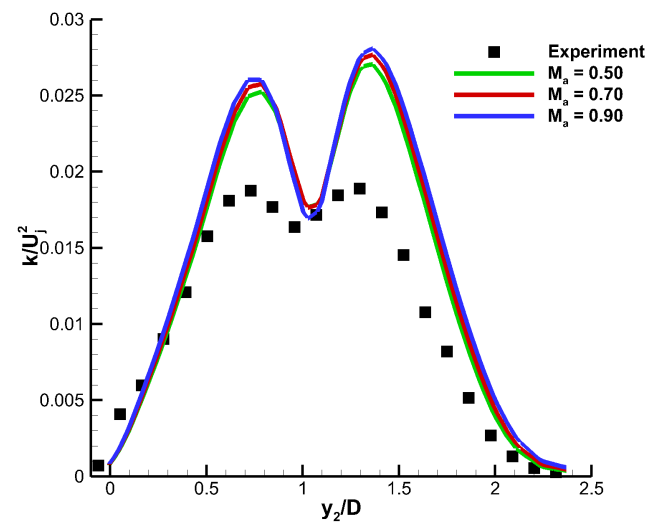

(b). Turbulent Kinetic Energy (TKE), $k / U_{J}^{2}$

Figure 8- Comparison of normalized mean flow and TKE obtained by RANS SolidWorks ${ }^{\circledR}$ calculation at the trailing edge location, $y_{d} / D_{J}=1.2, x_{d} / D_{J}=5.7, D_{J}=2.12 "$, with low Mach number \& high aspect ratio experiment reported in Zaman [6], where jet Mach number is Ma $=0.22$ and $A R=8$.

The results in Figure 8 show that, while the shapes of the axial velocity and turbulent kinetic energy distributions are well predicted, the peak TKE is over-predicted by about $25 \%$ compared to PIV data at the trailing edge location. Nonetheless, these differences are consistent with other RANS CFD codes, such as the NASA WIND code [19]. Cross flow distributions of mean axial velocity and turbulent kinetic energy, shown in Figure 9, near the surface trailing edge $\left(x_{d} / D_{J}=5.7\right.$ in Figure 4$)$, compare favorably with the experimental results.

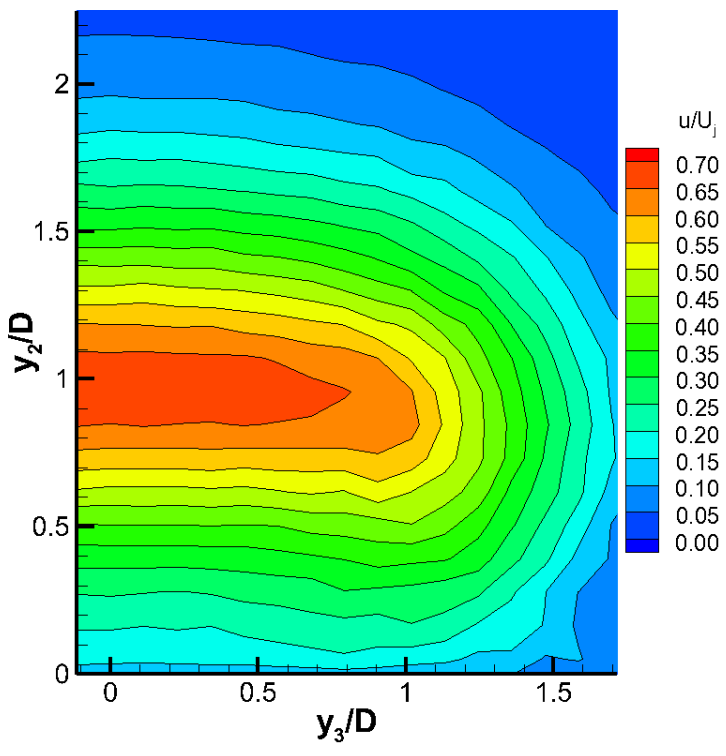

(a). $U / U_{J}$ : Zaman et al. [6].

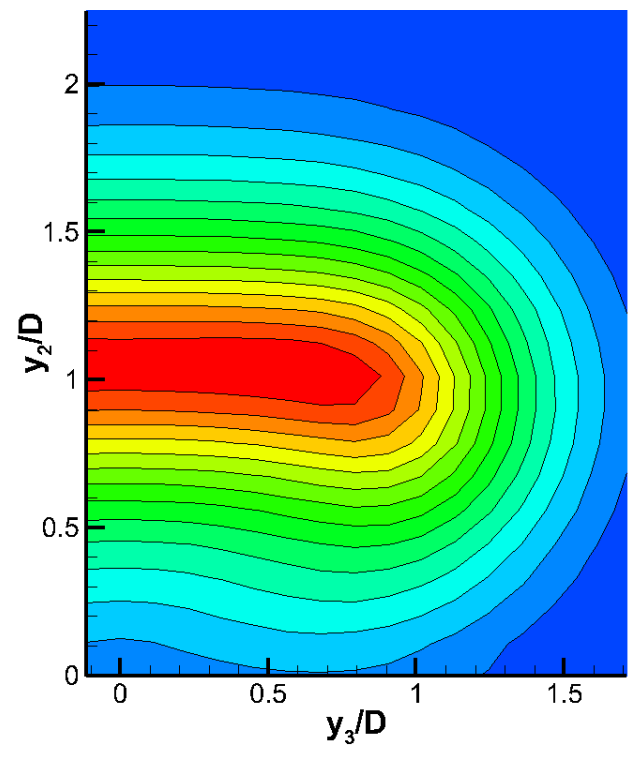

(b). $U / U_{J}:$ RANS calculation. 


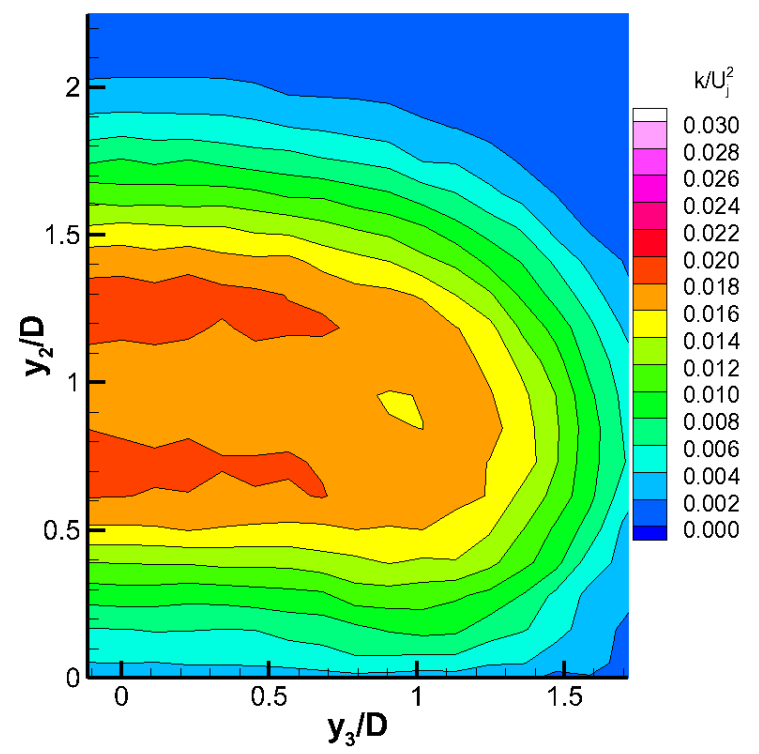

(c). TKE, $k / U_{J}^{2}:$ Zaman [6].

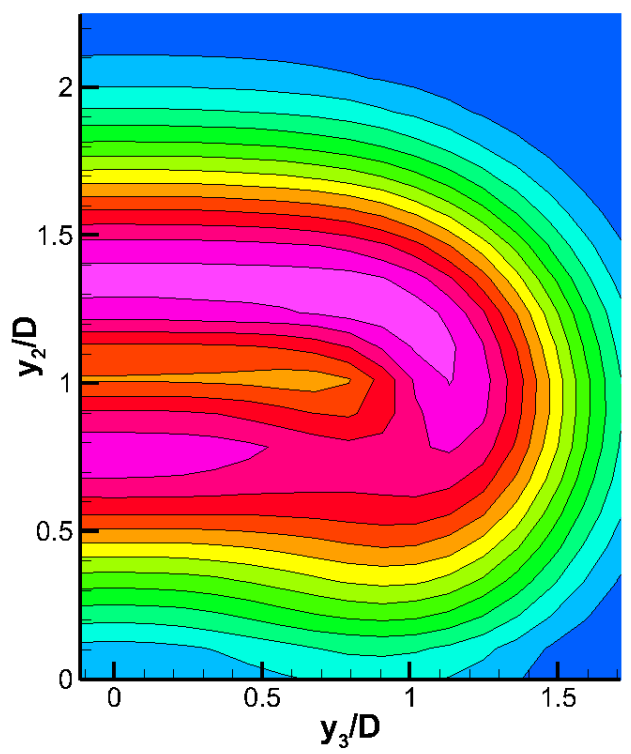

(d). TKE, $k / U_{J}^{2}$ : RANS calculaton.

Figure 9 - Comparison of Normalized mean velocity and TKE obtained by RANS SolidWorks ${ }^{\circledR}$ calculation and experiments reported in Zaman [6] for same trailing edge location as the caption in .

Figure 10 shows values of the normalized RANS-based TKE and length scale, $L_{R A N S}=k^{3 / 2} / \varepsilon$, at the trailing edge of the plate at the peak mean axial velocity location at the center of the span for $M a=0.5,0.7,0.9$ and $A R 2, A R 4, A R 8$. The results show that the RANS-based TKE and length scale reduce as the nozzle AR reduces for any given acoustic Mach number. On the other hand, the variation of these normalized scales is relatively insignificant at a fixed nozzle AR and varying acoustic Mach number.

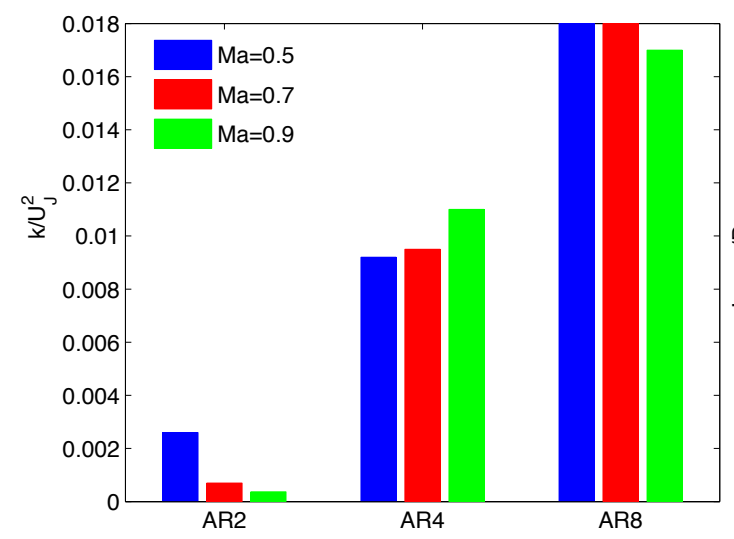

(a). TKE, $k / U_{J}^{2}$

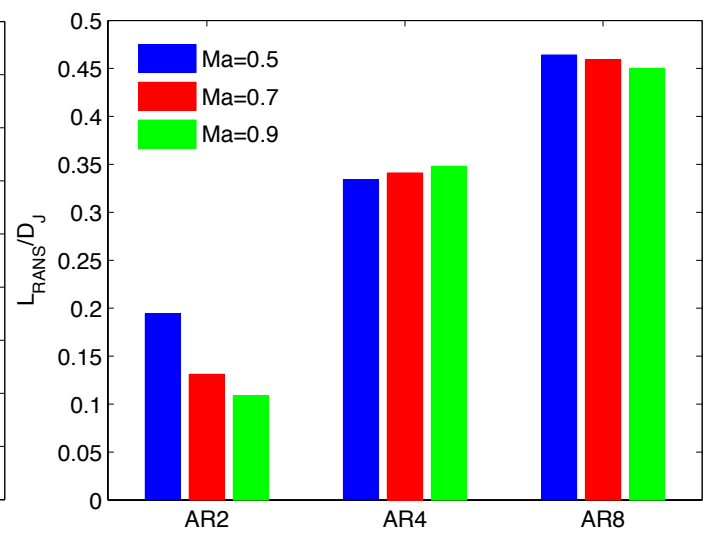

(b). RANS length scale, $L_{R A N S} / D_{J}$

Figure 10 - . Variation of normalized TKE and length scale obtained by RANS SolidWorks ${ }^{\circledR}$ calculation with Nozzle Aspect Ratio (AR) and Acoustic Mach number. 


\section{RANS-Based Jet-Surface Interaction Noise Predictions}

Using the RANS solutions described in the last section, we define the lengthscales to be $l_{n}=c_{n} k^{3 / 2} / \varepsilon, n=0,1$ and 3 and the amplitude $\Psi_{0}=c_{\Psi} \bar{\rho} k$ in the jet-surface interaction model, (28). Moreover, the mean flow $U / U_{J}$ is obtained directly from the RANS calculation at the trailing edge of the plate. The scaling coefficients are then tuned so that the predictions at polar angle, $\theta=90$ and $M a=0.9$ (where jet-surface interaction is greatest, see Figure 5 and Figure 7) and $A R 8$ for which the theory is directly applicable.

In Figure 11 and Figure 12 we show the RANS-based $\theta=90$ spectra for $M a=0.7$ and $M a=0.9$, respectively for aspect ratios $A R 4$ and $A R 8$. The low frequency roll-off is predicted well for almost all cases shown in Figures 11 and 12. There is a some underprediction of $\mathrm{Ma}=0.7$ and $\mathrm{AR}=4$. They are, however, encouraging given that the model parameters in (28) have been kept fixed at $\left(c_{0}, c_{1}, c_{3}\right)=(1.4,0.021,0.022), U_{c}=0.60 U_{d} ; b_{0}=0.6$ $c_{\Psi}=1.0$ and $\left(a_{0}, a_{1}, a_{2}\right)=(0.82,0.88,0.05)$ in all cases, with exception of the gspanwise length $L_{3} / D_{J}$, where $L_{3} / D_{J}=10$ for $\mathrm{AR}=4$ case and $L_{3} / D_{J}=20$ for $\mathrm{AR}=8$. The latter reflects the larger spanwise extent of the turbulence in the larger aspect ratio case.

It is expected that, with further experimentation with $c_{0}, c_{1}, c_{3}$ and $c_{\Psi}$,the predictions could, potentially, be made to agree better with the data, especially if the model parameters in (28) are also tuned for each nozzle aspect ratio.

We note that the model does not predict the oscillations that are present in the data at very low frequency. These are believed to be due to scattering of the jet noise by the edge of the plate, rather than being directly produced by the edge-noise source treated in this work.

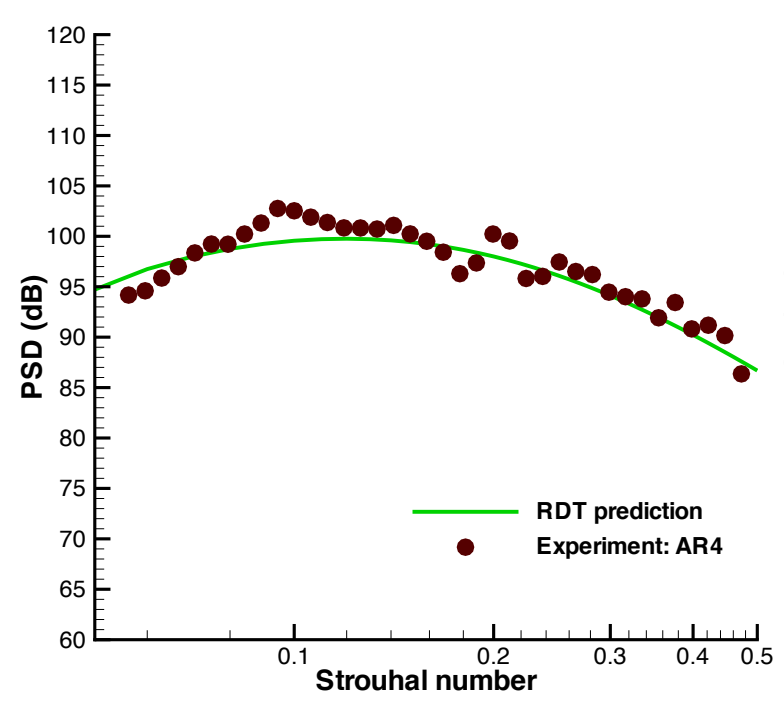

(a). $A R=4$

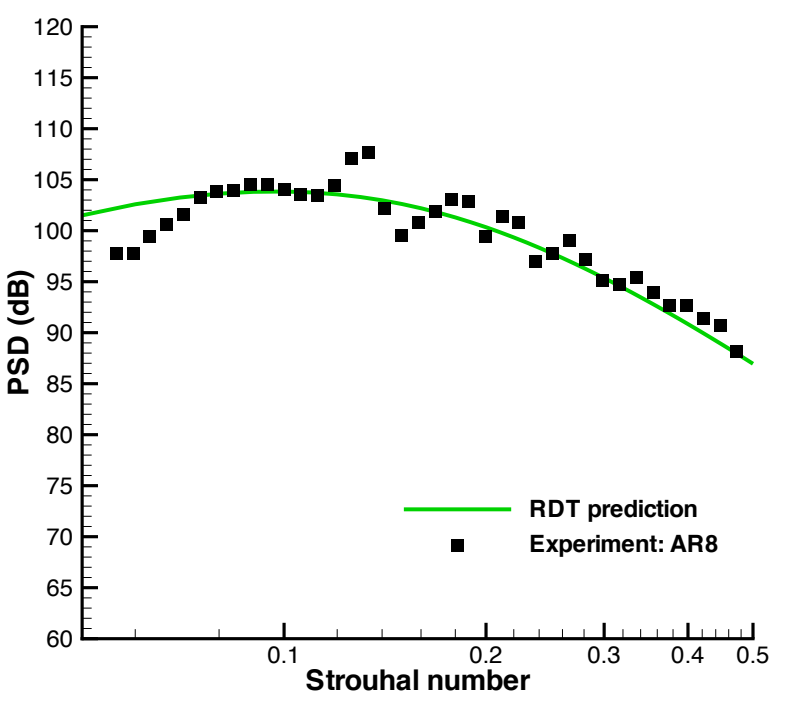

(b). $A R=8$

Figure 11 - $\theta=90^{\circ}$ spectrum: RDT prediction for $M a=0.9$ compared to noise data . 


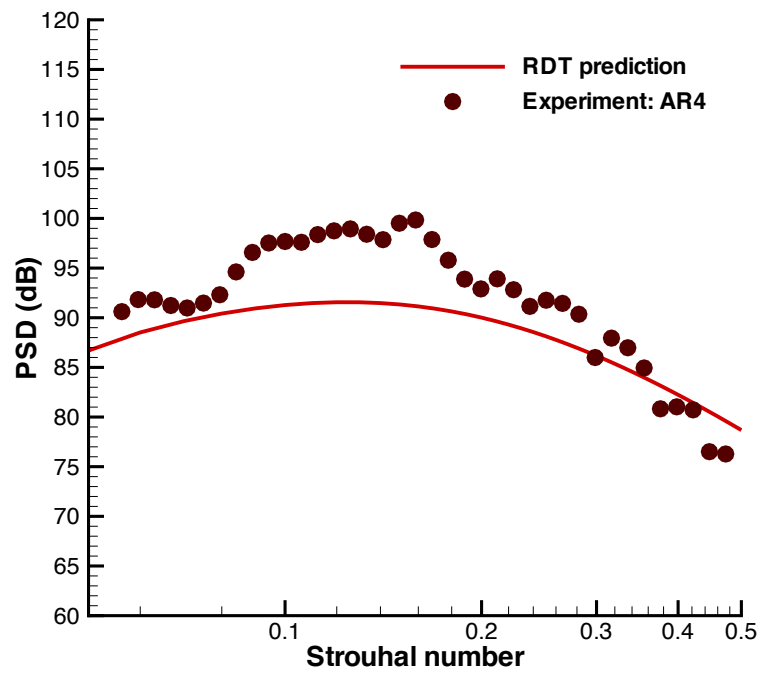

(a). $A R=4$

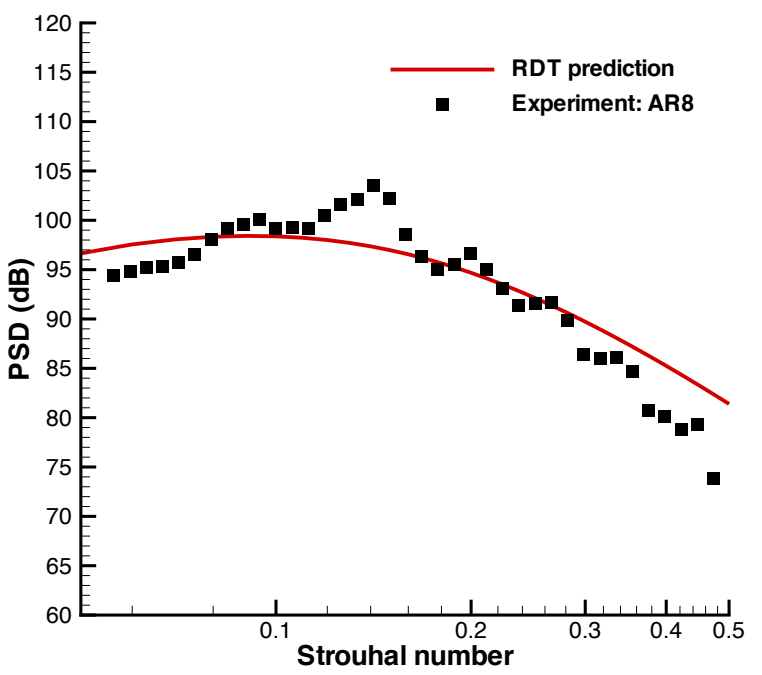

(b). $A R=8$

Figure 12 - $\theta=90^{\circ}$ spectrum: RDT prediction for $M a=0.7$ compared to noise data. Parameters same as in

\section{Conclusions and Future Work}

In this paper we have extended the jet-surface interaction model developed in [1], where it was shown that the jet-surface interaction noise spectrum, $I_{\omega}(\boldsymbol{x}),(28)$, at the observation point $\boldsymbol{x}$, is given by the integral of a directivity factor (24) and a source function, $S\left(y_{2}, \tilde{y}_{2} ; k_{3}^{(s)}, \omega\right)$, that is related to the upstream turbulence correlation, (25), by the algebraic correspondence relation (21). In this paper we have extended the GAL model to include a finite de-correlation region in the upstream turbulence correlation function (25) by introducing the turbulence model (26)which exhibits properties of type shown in Figure 3 (the auto-correlation of (25)). We have shown, using simple asymptotic arguments and numerical analysis, that the presence of a de-correlation region (i.e. taking $a_{1}>0$ in(26)) directly affects the low-frequency algebraic decay of the jet-surface interaction noise spectrum. This decay, often termed the low frequency 'roll-off', must be $O\left(\omega^{2}\right)$ for the acoustic field to be consistent with the dipole-like measured 'roll-off' in the experiments reported in Bridges [8]. A finite de-correlation is required for the roll-off to be $O\left(\omega^{2}\right)$ at very low frequencies where the maximum sound amplification occurs. In constrast, the GAL model

(eq. 6.50 in that paper) did not include the de-correlation effect and thereby produced a spectrum that tends to $O(1)$ at very low frequencies, which is at worst $10 \mathrm{~dB}$ greater than experiment (see figure $5 \mathrm{~b}$ ).

The model we have used in this paper, (29), also gives predictions that are reasonably accurate for more threedimensional flows associated with lower aspect ratio rectangular jets (Figure 7). In addtion we have implemented a RANS-based RDT prediction method that takes into account the reduction in length scales and turbulent kinetic energy with nozzle aspect ratio predicted by these flow solutions. This approach generally gives predictions within experimental uncertainty. In principle, any empiriscim introduced by tuning the scales from RANS calculation could be eliminated by using experimental or LES data on turbulence (as, for example in [18]). 
Professor J. T. Stuart ${ }^{4}$ recently pointed out that the importance of any noise prediction model is in its ability to show how to reduce the far-field noise. The jet-surface interaction model (28) based on the non-homogeneous rapiddistortion theory (RDT) allows the "exact" turbulence conditions to be specified as its upstream boundary condition through the algebraic relation (21) that is a function of the two-point time-delayed correlation (25) of the stationary

random function $\rho v_{\perp}^{\prime}(\boldsymbol{x}, t)$ (transverse momentum fluctuation), which Tennekes \& Lumley [13] explained must go negative with increase in spatial separation and/or time delay in a time-stationary turbulence field. The parametric study in figure 4.1 a shows that varying the de-correlation region (increasing coefficient, $a_{1}$ ) varies the predictions so that they lie between GAL model $\left(a_{1}=0\right)$ to the present results (figure 4.1b) which possesses the correct $O\left(\omega^{2}\right)$ low frequency roll-off. In principle, however, further increases in $a_{1}$, could provide a means to reduce the low frequency amplification associated with jet-surface interaction. Physically, this condition would indicate the presence of larger, more-organized, structures within the turbulence that are more likely to de-correlate than finerscale turbulence.

\section{Appendix}

Equation (22) shows that $E\left(y_{2} ; k_{3}^{(s)}, \omega\right)$ expands as

$$
E\left(y_{2} ; 0, \omega\right) \equiv \frac{1}{c_{\infty}^{2}}\left(\left[U\left(y_{d}\right)-U\left(y_{2}\right)\right]+i \omega S\right)+O\left(y_{2}-y_{d}\right)
$$

since $U\left(y_{2}\right)=U\left(y_{d}\right)+O\left(y_{2}-y_{d}\right)$ and $c^{2}\left(y_{2}\right)=c_{\infty}^{2}+O\left(y_{2}-y_{d}\right)$ when $y_{2}=O\left(y_{d}\right)$, where $S\left(y_{d}\right)$ is given by

$$
S\left(y_{d}\right)=\frac{\pi c_{\infty} U_{d} b_{0}}{U^{\prime \prime} y_{d} \sqrt{c_{\infty}^{2}-U_{d}^{2}}}
$$

Integral (29) then expands as

$$
\begin{aligned}
& I_{0}(M a, \theta, \pm \pi / 2)= \\
& \qquad 4 \frac{c_{\infty} M a^{3} \tilde{\Pi}\left(\omega / U_{d}, \omega, 0\right)}{(1-M a \cos \theta)^{2}(1-M a)} \int_{0}^{M a} \frac{1}{\left|E\left(y_{2} ; 0, \omega\right)\right|^{2}} d M\left(y_{2}\right), \quad \text { for } y_{2}=O\left(y_{d}\right)
\end{aligned}
$$

where $M a=M\left(y_{d}\right)$ and $U_{d}=U\left(y_{d}\right)$. Inserting (33) result into this latter integral shows:

\footnotetext{
${ }^{4}$ Private communication.
} 


$$
\begin{aligned}
& I_{0}(M a, \theta, \pm \pi / 2) \approx \\
& \quad 4 \frac{c_{\infty} U_{d}^{3} \tilde{\Pi}\left(\omega / U_{d}, \omega, 0\right)}{(1-M a \cos \theta)^{2}(1-M a)} \int_{0}^{U_{d}} \frac{1}{\left[U\left(y_{d}\right)-U\left(y_{2}\right)\right]^{2}+(\omega S)^{2}} d U\left(y_{2}\right), \\
& =4 \frac{c_{\infty} U_{d}^{3} \tilde{\Pi}\left(\omega / U_{d}, \omega, 0\right)}{(1-M a \cos \theta)^{2}(1-M a)} \frac{\pi}{2 \omega S}, \quad \text { for } y_{2}=O\left(y_{d}\right)
\end{aligned}
$$

The integral in (36) is now $O(1 / \omega)$ where $S=O(1)$. Given that equation (31) shows $\chi\left(\omega / U_{d}, 0\right) \rightarrow 1$ as $\omega \rightarrow 0$, the function, $\tilde{\Pi}\left(\omega / U_{d}, \omega, 0\right)$ (using (30)) expands as,

$$
\tilde{\Pi}\left(\omega / U_{d}, \omega, 0\right) \sim\left\{\begin{array}{c}
1, \text { for } a_{1}=a_{2}=0 \\
\omega^{2}, \text { for } a_{0} \sim a_{1}
\end{array}\right.
$$

and, therefore, integral (29) possesses asymptotic properties:

$$
I_{0}(M a, \theta, \pm \pi / 2) \sim\left\{\begin{array}{c}
1 / \omega, \text { for } a_{1}=a_{2}=0 \\
\omega, \text { for } a_{0} \sim a_{1}
\end{array}\right.
$$

when $\omega \rightarrow 0$.

\section{Acknowledgments}

MZA would like to thank financial support from Chapman Fellowship (2013-2014) at Imperial College London, Department of Mathematics and would like to thank useful discusions with Professor W. Devenport at Virginia Tech. The work was also supported by the NASA Fundamental Aeronautics Program, High Speed Project.

\section{References}

[1] Goldstein, M. E., Afsar, M. Z. \& Leib, S. J., "Non-Homogeneous Rapid Distortion Theory on Transversely Sheared Mean Flows," Journal of Fluid Mechanics, vol. 736, 2013, pp. 532-569.

[2] Olsen, W. and Boldman, D., "Trailing edge noise data with comparison to theory," AIAA Paper 79-1524, 1979.

[3] Wang. M.E., Wing effect on jet noise propagation,' J. Aircraft ,Vol. 18, No. 4, pp. 295 - 302, 1981.

[4] Brown. C., 'Jet-surface interaction test: far-field noise results,' GT2012-69639: ASME IGTI Turbo Expo, June 11-15, 2012

[5] Bridges, J.E., Brown, C. A. and Bozak, R., "Experiments on Exhaust Noise of Tightly Integrated Propulsion Systems" AIAA Paper 2014-2904, 2014.

[6] Zaman, K. B. M. Q., Brown, C. A.; Bridges, J. E., "Interaction of a Rectangular Jet with a Flat-plate Placed Parallel to the Flow," AIAA Paper 2013-2184, 2013. 
[7] Zaman, K. B. M. Q., Fagan, A., Clem, M and Brown, C. A., "Resonant Interaction of a Rectangular Jet with a Flat-plate," AIAA Paper 2014-0877, 2014.

[8] Bridges, J., “ Noise from Aft Deck Exhaust Nozzles - Differences in Experimental Embodiments,” AIAA Paper 2014-0876, 2014.

[9] Brown, C., "Developing an Empirical Model for Jet-Surface Interaction Noise," AIAA paper 2014-0878, 2014.

[10] "Advanced Boundary Cartesian Meshing Technology in SolidWorks Flow Simulation" Dassault Systemes SolidWorks Corporation Technical Paper.

[11] "Enhanced Turbulence Modelling in SolidWorks Flow Simulation" Dassault Systemes SolidWorks Corporation Technical Paper.

[12] Leib, S.J. and Goldstein, M.E., "Hybrid Source Model for Predicting High-Speed Jet Noise," AIAA Journal, Vol. 49, No. 7, pp. 1324-1335, 2011

[13] Tennekes, H. and Lumley, J., A first course in turbulence, MIT Press, Cambridge, Mass. 1971.

[14] Goldstein, M.E., "Scattering and Distortion of the Unsteady Motion on Transversely Sheared Mean Flows, J. Fluid Mech. Vol. 91, pp. 601-632,1979.

[15] Goldstein, M. E., Aeroacoustics, McGraw Hill, New York, 1976.

[16] Bridges, J, and Brown, C.A., "Validation of the small Hot jet Rig for Jet Noise Research," AIAA Paper 2005-2846, 2005.

[17] Afsar, M.Z., Leib, S. J. and Bozak, R.F., "Low frequency roll-off of the jet-surface interaction noise spectrum in high-speed rectangular air jets," To be submitted to J. Sound and Vib.

[18] Karabasov, S.A., Afsar, M.Z., Hynes, T.P., Dowlong, A.P., McMullan, W.A., Pokora, C.D., Page, G.J. and McGuirk, J.J, "Jet Noise: Acoustic Analogy Informed by Large Eddy Simulation," AIAA Journal, Vol. 48, No. 7, pp. 1312-1325, 2010.

[19] Dippold, V., "Acoustic Reference Nozzle with Mach 0.97, Unheated Jet Flow," NPARC Alliance Validation Archive, NASA Glenn Research Center, http://www.grc.nasa.gov/WWW/wind/valid/arn/index.html 